\title{
Agrippa und Boerhaave: Zwei Positionen im Ringen um die certitudo medicinae
}

\author{
Von Fridolf Kudlien, Kiel
}

Niemand wird bezweifeln, daß die «Gewißheit» ein zentrales geistiges Problem auch der sich entwickelnden Medizin ist. In der Tat: «Alle versuchten und mißglückten Wege zu ihr aufzudecken, hieße eine Geschichte der Medizin unter einem vieles erhellenden Gesichtspunkt schreiben ». ${ }^{1}$ Ebensowenig wird man jedoch daran zweifeln, daß hier eine für einen einzigen Historiker wohl kaum zu bewältigende Aufgabe gestellt ist - selbst wenn es sicherlich nicht zutrifft, daß «alle medizinische Forschung seit der Antike im Zeichen des Ringens um diese Gewißheit steht ». ${ }^{2}$

Wir möchten ERNa LeSKys Anregungen fortsetzen, indem wir an zwei typischen Beispielen zwei grundverschiedene Positionen im Ringen um die certitudo medicinae deutlich zu machen suchen. Damit hoffen wir, den gemeinten geistigen Prozeß ein wenig weiter ins Helle zu rücken und Kundigeren den Weg zu einer lockenden Aufgabe wissenschaftshistorischer Forschung zu ebnen.

Es soll sich dabei auch zeigen, daß CABANIS' berühmte und einflußreiche Schrift Du degré de certitude de la médecine in gewissem Grade in einer ausgeprägten und weit über die besondere kritische Situation der Medizin um 1790, über Destutt de Tracy und Condillac zurückreichenden geistigen Tradition steht. Uns scheint, daß die «Renaissance» genannte geistige Bewegung des 16.Jahrhunderts und die durch sie auf den Weg gebrachten naturwissenschaftlichen Entwicklungen das Janusgesicht einer naturwissenschaftlich-medizinischen «Gewißheit» recht eigentlich wiederentdeckt haben. Lenken wir also zunächst den Blick auf einen bestimmten Punkt in jener vielschichtigen und widersprüchlichen Epoche.

Im Jahre 1530 veröffentlichte Heinrich Cornelius Agrippa von NetTesheim, Stadtarzt von Beruf, eine Schrift mit dem provozierenden Titel De incertitudine et vanitate scientiarum. Sie trägt obendrein den Untertitel «declamatio invectiva»; ihre wissenschaftsfeindliche Tendenz ist damit von vornherein klargestellt. Die uns hier allein interessierende Medizin handelt Agrippa ab in den Kapiteln LXXXII-LXXXVI und LXXXVIII un-

1 E.Lesky, Cabanis und die Gewißheit der Heilkunde, Gesnerus 11 (1954) 155.

${ }^{2}$ Siehe Lesky, a. $O$. 
ter den Überschriften «de medicina in genere; de medicina operatrice (d.i. praktische Medizin); de pharmacopolia; de chirurgia; de anatomistica; de diaetaria ". ${ }^{3}$

Was hat Agrippa an der Medizin als scientia auszusetzen? Wir müssen zwei Aspekte seiner Kritik unterscheiden: den ausgesprochen satirischen und den ernsthaft-grundsätzlichen. Zu den rein satirischen Tönen gehört etwa, daß die Medizin anschließend an das Militärwesen erörtert wird, weil auch sie «quaedam homicidiorum ars» sei! Oder etwa folgendes «Rezept»: «Nimm einen Notar, füge nach Bedarf einen Priester mit Weihwasser und Öl hinzu und bereite alles in Deinem Hause zu - weil Du sterben wirst!» Dies allein schon dürfte an Bekannteres aus der Weltliteratur erinnern, nämlich MolıÈres Ärzteverspottung. Noch mehr gilt das für die Stelle, wo Agrippa beklagt, daß der Kranke dem Arzt einfach ausgeliefert sei: Die Ärzte schuldigen bei letalem Ausgang frech alle möglichen Umstände an nur nicht ihre eigene Unfähigkeit; sie fordern gar noch Honorar für einen Mord, der ohne sie gar nicht passiert wäre. Und das alles mit ruhigem Gewissen, «cum quia error eorum terra tegitur tum quia irremeabilis est mortuorum regio». Das steht genauso bei Molière, etwa im Arzt wider Willen, III 1 (übersetzt A. Luther), wo Sganarell sagt «... hier aber kann man einen Menschen umbringen, ohne daß es einen was kostete. Wir haben uns nie geirrt, die Schuld trägt immer der Verstorbene. Und das Beste an diesem Beruf ist eben, daß unter den Toten eine Diskretion, eine Ehrbarkeit herrscht, wie sonst nirgends in der Welt: noch nie hat sich einer über den Arzt beklagt, der ihn umgebracht hat.»

Läßt man diese satirischen Töne einmal beiseite, dann geht Agrippas Vorwurf der incertitudo medicinae auf folgendes: Die theoretische Basis der Medizin sei seit der Antike denkbar unsicher; schon bei den alten Ärzten streiten ständig die verschiedensten Meinungen gegeneinander - wie bei den Philosophen. Das führt Agrippa am Beispiel der Samentheorien im einzelnen aus. Die praktische Medizin ruht ebenfalls auf einer ganz unsicheren Basis, nämlich auf fallacia experimenta. Den Begriff «experimentum » wird man hier sowohl als «Erfahrung» wie auch als «Experiment» nehmen müssen, wie schon im Mittelalter etwa bei Roger Bacon ${ }^{4}$; zweifellos ist die letztere Bedeutung gemeint, wenn Agrippa von «experimenta pericu-

${ }^{3}$ Ich benutze die Ausgabe Köln 1575, welche unpaginiert ist; daher kann ich nur auf die Kapitel als Ganzes verweisen.

4 Vgl. dazu H. Fischer, Arzt und Humanismus, Zürich 1962, S. 89. 
losa » spricht. Aus der Unsicherheit ihrer Basis folgert Agrippa für die praktische Medizin: «nec quicquam apud eos (sc. medicos) certi, sed omnia promissa eorum nugae volatiles et mera mendacia». Schließlich nimmt Agrippa noch Chirurgie und Anatomie mit zwei besonderen Vorwürfen aufs Korn: Ihre Vertreter seien carnifices, ihr Tun sei «blutige Grausamkeit» und «unfromm ".

Die Frage nun, wie die dezidierte Wissenschaftsfeindlichkeit Agrippas geistesgeschichtlich zu deuten sei, ist erst neuerdings wieder angeschnitten worden. Rudolf Schmitz schreibt dazu ${ }^{5}$ : «Für viele ist Agrippa ... der Vertreter einer in Deutschland sonst nicht vorkommenden Renaissance italienischer Prägung ... Der näherliegende Gedanke, ihn lediglich als Prototyp der Menschen seiner rheinischen Heimat zu sehen, die Möglichkeit, deren Spott- und Kritisierlust ... für die Niederschrift der ,Eitelkeiten ' verantwortlich zu machen, wurde noch nie berücksichtigt. Auf diese Weise aber wäre man imstande, manches Ungereimte zu erklären.»

Ich glaube, daß eine kurze Analyse der Vorwürfe, die Agrippa der Medizin macht, ausreicht, um den geschichtlichen Standort der «Eitelkeiten» einigermaßen zu bestimmen. Nehmen wir zunächst die «Unfrommheit» (sie gilt bei Agrippa übrigens auch für die Diätetik, wegen ihrer Verzerrung der gottgeschaffenen Lebensweise): Das ist nicht nur eine traditionelle Formel, sondern gehört zur geistigen Grundhaltung des Agrippa, die sich besonders unmißverständlich in der Vorrede zu den «Eitelkeiten » ausdrückt. Dort wird als Motiv für seine Schrift ausdrücklich nicht Haß, Ehrgeiz oder ähnliches genannt, sondern der Eindruck, daß «viele sich an die humanae disciplinae derartig hängen, daß die Kenntnis der Heiligen Schriften (welche zwar ohne rhetorischen Schmuck, dafür aber auf Tugendübung und reinen Glauben gegründet sind) nicht nur verschmäht, sondern sogar mit Verachtung verfolgt wird». Das nennt Agrippa ohne Umschweife ein Abirren von der Erkenntnis der Wahrheit.

Nachdem wir das gehört haben, werden wir Agrippa nicht mißverstehen, wenn er sagt, es sei eine impia tyrannis, die Studierenden auf auctores festzulegen und ihnen damit die Freiheit zu nehmen, von sich aus der Wahrheit nachzuspüren. Aus dem Zusammenhang gerissen, klingt so etwas wie ein souveräner Angriff aus humanistischem Geiste gegen die mittelalterliche scholastische Lehrmethode, und es ist tatsächlich auch ein Angriff dagegen,

5 In seinem Aufsatz «Magie und Substanz bei Agrippa von Nettesheim», Hippokrates 35 (1964) 651. 
aber eben nicht aus humanistischen Motiven: die Wahrheit - das ist hier eindeutig Gottes Wort. Agrippa bestätigt uns, es sei eine «arrogans praesumptio», die menschlichen Meinungen - also die gesamte Wissenschaft dem Worte Gottes gleichzuachten oder sogar vorzuziehen.

Agrippas Wissenschaftsfeindlichkeit entstammt also seiner religiösen Haltung. Die Wissenschaften müssen ihm «unsicher», «nichtig» sein, sie können in nichts anderem als zweifelhaften menschlichen «opiniones » bestehen, weil sie den Boden der Heiligen Schrift, der Beschäftigung mit Gottes Wort verlassen haben. Wenn wir nun noch Agrippas weitere Vorwürfe gegen die Medizin - Grausamkeit und Vertrauen auf trügerische "experimenta»zur «Unfrommheit» hinzunehmen, dann liegt der unmittelbare Quellbezirk seiner Wissenschaftsfeindlichkeit offen: Diese ergibt sich aus seinem christlich getönten Platonismus.

Wie uns vor allem O. Regenbogen gezeigt hat ${ }^{6}$, sind die Angriffe PlaToNs gegen die rein empirische Naturwissenschaft ionischer Prägung bisweilen geradezu in priesterlich herablassendem Ton gehalten. Die Platonische Ablehnung des Experiments verwendet typischerweise das Wort $\sigma \tau \varrho \varepsilon \beta \lambda o \tilde{v}$ «martern, foltern, unnütz quälen»; das ist einer der Ursprünge des späteren «crudelitas»-Vorwurfs gegen Anatomie und Physiologie. Dazu kommt Platons Hinweis auf die Unzulänglichkeit menschlicher Sinneswahrnehmungen und menschlichen Urteilsvermögens und (etwa im Timaios) der Vorwurf, solche empirisch-experimentelle Forschung verkenne die Grenze zwischen göttlichem und menschlichem Wissen - sie ist also «unfromm»!

Man sieht, wie Agrippas Vorwürfe, etwas vergröbert, den Platonischen entsprechen. Er ist Platoniker, und er ist gleichzeitig Christ; das Etikett «Neuplatonismus» und der Name Marsilio Ficinos werden also mit Recht bei der Bestimmung von Agrippas geistigem Standort verwendet. Die Zwiespältigkeit Agrippas, seine Wissenschaftsfeindlichkeit und dann wiederum seine überraschende Astrologiegläubigkeit entspricht genau FICINos eigener zwiespältiger Haltung etwa gegenüber der Astrologie ${ }^{7}$. Wir werden hinzufügen dürfen, daß das alles auch unter der allgemeinen Spannung zwischen christlichem Glauben und «Renaissance», d.h. Wiedererweckung der heidnischen Antike, gesehen werden muß. Es gab da zwei ex-

${ }^{6}$ Kl. Schriften, München 1961, S. 163-165 (mit den notwendigen Belegen).

7 Dazu K.Sudhoff, Iatromathematiker vornehmlich im 15. und 16. Jahrhundert, $A b h$. Gesch.Med.2 (1902) $31 \mathrm{f}$. 
treme Gegenpole und dazwischen viele Nuancen. Agrippa hat den Standpunkt des "radikalen» Christen, das bedeutet der Feindschaft gegen die Wissenschaft als heidnisches Erbe, eingenommen. Daß andererseits seine Vorwürfe gegen die Medizin, so grob sie sind, manche mittelalterlichen und zeitgenössischen Zustände tatsächlich treffen, ist klar. Die maßlose Übersteigerung seiner Kritik der Medizin deutet wiederum eigentümlich auf Molières (ansonsten viel «säkularisiertere») Ärztekritik voraus (siehe unten).

Ehe wir nun ein paar Worte zu der weiteren Entwicklung sagen, wollen wir einen Sprung vorwärts in die Zeit um 1700 tun. Daß der große BoerHAAVE seine erste Leydener Rektoratsrede am 8.Februar 1715 über das Thema «De comparando certo in physicis » gehalten hat (gedruckt im gleichen Jahr in Leyden bei Peter van der AA) ist gewiß kein Zufall. Damals war offenbar wieder ein Übelstand besonders manifest geworden, den Boerhaave gleich zu Beginn klar konstatiert (S.2): Die Menschen liebten es, naturwissenschaftliche Forschung nur theoretisch-spekulativ (sola meditatione) zu betreiben und dabei möglichst gleich etwas Endgültiges über die Grundkräfte des Universums auszusagen. Dieser Hang sei eine corruptela communis und verursache den meisten Schaden in der Medizin und für deren aktuelle Aufgaben. «Die Grundkräfte oder -ursachen der Dinge sind uns vollkommen verborgen. Wir können nur mit Hilfe der Sinneswahrnehmungen deren Wirkungen in Erfahrung bringen oder sie mit sicherer geometrischer Methodik feststellen.» Einige Seiten weiter (S.6): Wer die principia (Boerhaave nennt sie auch causae universales) zu kennen oder erkennen zu können behauptet, «strebt in ungeheurer, frevelhafter Torheit nach Gottes Weisheit». Die legitime Aufgabe der Forschung sei es (S. 7-10), sich wie die alten Philosophen - in einer Linie werden Le UKipp, DeмокRIt, Gassendi, Descartes und Newton genannt - an das «wahrhaft in Erfahrung Gebrachte» zu halten, etwa an die Atome, das Leere und das Phänomen des Gewichts. Damit käme man zwar nicht an die causae universales heran, aber doch an die causae proximae, d.h. an die den Sinneswahrnehmungen zugänglichen Wirkungen der «Prinzipien»: «Non ex causa effectum, sed ex hoc aliquid illius subintelligis.»

Hier wollen wir kurz auf Parallelen zu Cabanis' Argumenten ${ }^{8}$ aufmerksam machen: Auch Cabanis gibt zu, daß die Grundursachen und -kräfte (la force spontanée) unbekannt sind und bleiben; auch er begnügt sich mit

8 «Du degré...», S.51f.; siehe LeSKy, a. O., S.158. 
den Wirkungen der cause première, und er nennt ebenfalls als legitime Aufgabe die genaue und ständige Beobachtung der Phänomene (d.h. eben der Wirkungen); allein darauf gründen sich die Schlußfolgerungen. Boerhaaves «geometrische Methode» schätzt Cabanis freilich nicht («Du degré...», S. 87; vielleicht in Erinnerung an die Auswüchse der sogenannten Iatrophysik?). Wenn aber Boerhaave in der exakten geometrischen Methode Sicherheit auch für die Medizin finden wollte, dann ist das nicht nur typisch für die Zeit um 1700, wo man in allen möglichen natur- und geisteswissenschaftlichen Bereichen mit "geometrischer Methode» nach Sicherheit strebte (vgl. Spinozas Ethik!), sondern das knüpft an die Antike an: Niemand anders als GaLen ist es gewesen, dem nach eigenem Bekenntnis (Scr.minora, II 116f.) die exakte Methode der Geometrie und Arithmetik aus der lähmenden medizinischen Skepsis seiner Zeit heraushalf. Und die Unterscheidung der causae proximae von den causae primae oder universales, die Boerhaave ebenfalls mit Cabanis gemein hat ${ }^{9}$ ? Auch sie greift in

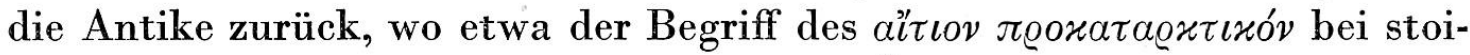
schen und skeptischen Philosophen und bei Ärzten (vgl. Galens Schrift De causis procatarcticis) dem $\pi \varrho \tilde{\omega} \tau o v$ ăı $\tau o v$ des Aristoteles gegenübergestellt wurde $^{10}$.

Im übrigen ist es sehr aufschlußreich, wie Boerhaave mit dem Vorwurf der «Ketzerei», der unfrommen und anmaßenden Einmischung in die Domäne rein göttlicher Weisheit operiert: Während dieses Platonische Gegenargument gegen die experimentelle Naturwissenschaft dem christlichen Neuplatoniker Agrippa als ein tödlicher Pfeil gegen die theoretische Medizin, vor allem die Anatomie, und damit gegen die gesamte Heilkunde diente, hat es bei Boerhaave seine Radikalität verloren, und zwar, wie man sieht, durch die (antike) Unterscheidung verschiedener Ursachengattungen. Es richtet sich nun nur noch gegen die Erforschung eines bestimmten Bereichs.

Wir fahren nun fort, die Boerhaaveschen Argumente zu referieren (a.O., S. 13) : Die Existenz des Leeren - d.h. einer der causae proximae - mechanisch bewiesen zu haben, sei eine große Leistung; aber daraus lasse sich dessen eigentliches principium, also die Ursache für das Leere, kaum ableiten. Ebenso stehe es mit der Schwerkraft. Boerhaave lobt ausdrücklich

9 Siehe für diesen LESKY, a. O., S.159f.

10 Vgl. auch meinen Beitrag «Funktionelle und deskriptive Anatomie in der Antike», Mitt. Inst.Gesch.Med. (Kiel) 1963, Heft 2, S.6, und meine Studie «Herophilos und der Beginn der medizinischen Skepsis», Gesnerus 21 (1964) $4 \mathrm{f}$. 
diejenigen, die, wie Huygens, Leibniz und Newton, «nicht erröten», solche Begrenztheit der Erkenntnismöglichkeiten offen zuzugeben ${ }^{11}$.

Nachdem er noch einmal das verfehlte Suchen nach den causae primae gebrandmarkt hat (S. 19: «caecutimus omnes pariter, quoties causas primas conamur intelligere»), lobt Boerhaave sodann ausführlich die Detailforschung. Er preist (S. 25) die medizinischen Entdecker von «causae proximae»: Drelincourt, Harvey, Malpighi, Swammerdam, de Graaf, Leeuwenhoek. Ein gutes Beispiel für solche Forschung seien die ova; sie sind so "gewiß» (firmis argumentis adstructa), daß nun keiner mehr an ihrer Existenz zweifeln könne. Jetzt käme es darauf an, jedes Einzelding in gleicher Weise zu erforschen (S. 28); nur so ließe sich Gewißheit gewinnen - nur einzeln würden die Dinge «fido naturae experimento» erkennbar (S. 29).

Für dieses im vollen Bewußtsein einer naturwissenschaftlichen Krise entworfene Forschungsprogramm gibt Boerhaave selber konkrete Aufgaben (S. 30 ff.): In der Anatomie sei etwa der Aufbau der Haut weiter zu untersuchen. Auch sei ein wichtiges Thema der Feinbau und die Physiologie des Haares (man solle bedenken, wie sehr die Haare offenbar mit dem Körper zusammenhingen). Von da aus leitet Boerhaave zu letzten zusammenfassenden Feststellungen über (S. $38 \mathrm{ff}$.): Auch der kleinste Einzelteil des menschlichen Körpers stünde in so vielfältigen Beziehungen zu allen übrigen Teilen, daß die Kenntnis der principia universalia (selbst wenn sie erreichbar wäre) für eine so konkrete Einzelforschung primär gar keinen Nutzen hätte ${ }^{12}$. Umgekehrt: Nur von exakt erforschten Einzeldingen aus könne die «fabrica» sichtbar gemacht werden. Die observatio accurata sei der wahre Weg. Das gelte genauso wie in der Geometrie und Mathematik: Descartes habe gerade mit solcher strikten methodischen Beschränkung die besten Ergebnisse für Geometrie, Mathematik und Mechanik erzielt, während bezeichnenderweise seine Physik und gar seine Physiologie nichts taugten, weil er hier nach den "principia» gesucht habe!

Indem Boerhaave so zum Ausgangspunkt zurückkehrt, schließt sich der Kreis seiner Rede. Wir können nun wenigstens andeutend den Weg markie-

11 Das erinnert daran, daß sich auch HARVEY voll bewußt war, man müsse zunächst einfach die Existenz des großen Kreislaufs (also einer «causa proxima »!) nachweisen (vgl. dazu meine «Herophilos»-Studie, S.12).

12 Genauso beantwortet CaBANIS das Argument, man müsse zuerst die principia universalia kennen, um medizinische Forschung und Praxis treiben zu können (siehe LeSKY, a. O., S.158). - Vgl. auch unten S.94. 
ren, der zwischen Agrippa und ihm und zwischen ihm und Cabanis liegt. Agrippa hatte mit seinem Angriff die Medizin (als eine der scientiae) schlechthin treffen und erledigen wollen. Wir haben schon festgestellt, daß das weit übers Ziel hinausschoß. Insofern eignet Agrippas Kritik auch gar keine effektive Wirkungsmöglichkeit, sondern nur eine charakteristische geistesgeschichtliche Symptomatik. Und in der Tat: In krassem Gegensatz zu Agrippas Wissenschaftsfeindlichkeit steht der geradezu hemmungslose naturwissenschaftliche Optimismus des fortschreitenden 16. Jahrhunderts, Erfahrung, «experimenta» gelten hier keineswegs als «fallacia». Empirische Tatsachenforschung, gegründet auf genaue Beobachtung, gewinnt schon im 16. Jahrhundert, lange vor dem Sensualismus Lockes und Humes, an Boden. Ein Anzeichen dafür ist die anwachsende «Observationes»-Literatur. (Wir erinnern nur an Namen wie Falloppio und Platter.) Auch die Philosophie betritt nun diesen Weg; ein Mann wie Bernardino Telesio (1508-1588) hat hier mit seiner sensualistisch-empiristischen Grundhaltung zweifellos eine ähnliche Rolle gespielt wie später Hume ${ }^{13}$.

Für diese Forschungsrichtung existiert also vom 16. ins 17. Jahrhundert ein kontinuierlicher Strom, der sich immer breiter entfaltet (Boerhaave nennt ja selbst die wichtigsten Namen dafür). Die Erfindung des Mikroskops hat dem natürlich weiteren Vorschub geleistet. Erst durch sie wurde die «Erforschung der kleinen Form », zu der Boerhaave so eindringlich aufruft, möglich und reizvoll.

Soviel über die Kontinuität einer auf Einzelforschung ausgerichteten, sensualistisch bestimmten Naturwissenschaft vom 16. über das 17. ins 18. Jahrhundert hinein. Wäre nun diese Richtung absolut vorherrschend ge-

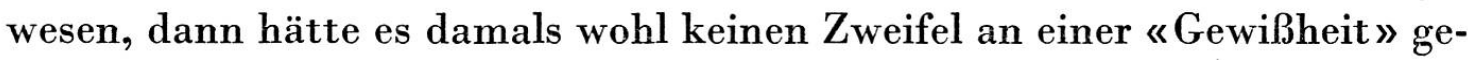
geben, jedenfalls nicht so, wie wir ihn als Hintergrund zu Boerhaaves Rede voraussetzen müssen. Was dieser als corruptela communis bezeichnet, war jedoch offenbar immer weiter als Gegenströmung wirksam: Es ist eben das alte Suchen nach den Grundprinzipien, den causae primae. Immer wieder wird dabei die Begrenztheit der menschlichen Erkenntnis schmerzlich bewußt. Das schafft dann immer von neuem Ungewißheit, Zweifel daran, ob eine sichere Erkenntnis überhaupt möglich ist, unter Umständen auch eine totale Skepsis. Für die Medizin erhebt sich etwa immer wieder die Frage: Können wir überhaupt Medizin treiben, wenn uns die letzten Ursachen des

13 Auf Telesio weist schon Neuburger (in Puschmanns Handbuch, Band II, S.14) im gleichen Zusammenhang kurz hin. 
Krankseins, die Grundprinzipien des Lebens unbekannt sind? Und immer wieder lautete die Antwort des «Positivisten»: Wir brauchen die Kenntnis der causae primae gar nicht, wenn wir uns in der Medizin in Selbstbeschränkung an das Erfahrbare halten.

Die Reaktionen auf das ständig wiederkehrende Problem der «Gewißheit» unterscheiden sich letzten Endes nur gradmäßig. Wenn wir uns etwa Molières Kritik an der Medizin des 17.Jahrhunderts ansehen (wie sie uns TemкIN ${ }^{14}$ analysiert hat), dann geht sie nicht, wie bei Agrippa, von religiösen Motiven aus - aber ihre Argumentation ist im Grunde die gleiche wie bei Agrippa, und genau wie dessen Kritik verliert sie im übersteigerten Anspruch den Boden unter den Füßen. Für beide gilt Temkins Feststellung (a.0., S. 67): «Was Molière vollbracht hat, ist eine große Auseinandersetzung mit einer Form der Medizin seiner Zeit, die ... sinnlos geworden war ... Als er aber weiterging, als er versuchte, die Medizin überhaupt anzugreifen, ... da ist er gescheitert.»

Boerhaaves Rede besaß also ihre volle Aktualität. Seine eigene Argumentation für die «Gewißheit» hatte, jedenfalls was die Medizin betrifft, durchaus Vorgänger: Schon Sydenham zum Beispiel, der Zeitgenosse Molières, antwortete auf die Zweifel daran, daß man Krankheiten überhaupt ohne Kenntnis der letzten Ursachen behandeln könne, mit den Worten: «Nicht mit Hilfe der Kenntnis der Ursachen, sondern mit Hilfe der Kenntnis einer angemessenen, durch die Erfahrung erhärteten Methode werden die meisten Krankheiten behandelt» (siehe Temkin, a.O., S. 82). Aber Boerhaaves im Grunde also gar nicht originelle Rede scheint durch ihre grundsätzliche, zusammenfassende, auf künftige Aufgaben hindeutende Behandlung des Themas «Gewißheit in den Naturwissenschaften» das größte Gewicht unter allen ähnlichen Verlautbarungen zu haben. Sie sah die Dinge ganz real: Die Erforschung der «kleinen Form», des einzelnen Teils hatte schon im 17.Jahrhundert die besten Erfolge gezeitigt und konnte im 18. Jahrhundert, soweit sie sich streng an diese selbstgewählte Beschränkung hielt, womöglich noch erfolgreicher sein.

Es lohnt, dies noch einen Augenblick lang weiterzuverfolgen: Der Boerhaaveschen Anregung zur Erforschung des Haares war im 18. Jahrhundert tatsächlich eine Reihe von Monographien über dieses Thema gefolgt; hier führte aber doch der Weg von exakter anatomisch-morphologischer For-

14 Studien zum «Sinn»-Begriff in der Medizin, Kyklos II (1929) 66-84 (Molière und der Sinn der Medizin im 17. Jahrhundert). 
schung wieder ab in die Höhen (oder Niederungen) der Spekulation; Boerhaaves eigentliches Anliegen mißachtend, übersteigerte man dieses Thema bei weitem in Richtung der causae primae ${ }^{15}$. Und noch auf etwas anderes wollen wir aufmerksam machen: Recht eigentlich nach Boerhaaves Rede kommt die «signa certa»-Literatur in der Medizin in Schwung. Wenn Boerhaave den Blick der Naturwissenschaftler von den causae primae weg auf deren effectus lenken wollte, so manifestieren sich solche effectus ja eben in nichts anderem als in signa. Lag es nicht also auf der Hand, im Streben nach der «Gewißheit» nach den «sicheren » unter den Anzeichen zu suchen, wie es die Antike schon getan hatte? Besonders das Problem des Scheintodes ist im 18. Jahrhundert unter diesem Aspekt bemerkenswert häufig untersucht worden ${ }^{16}$. Auch hier geht es freilich von anfänglichem Optimismus bis zu fast vollständiger Skepsis, wie sie sich etwa in K. Hinlys Commentatio super mortis historiam, causas et signa 1794 - wenige Jahre vor Cabanis' berühmter Schrift! - äußert.

Noch ein weiterer Hinweis zum 18.Jahrhundert: Wie Boerhaave die «certitudo» im Rahmen der gesamten Naturwissenschaft für die theoretische Medizin abgehandelt und in ihren Grenzen klargestellt hatte, so setzt sich sein Zeitgenosse Friedrich Hoffmann in der Medicina rationalis systematica (beginnt Halle 1718 zu erscheinen) für die praktisch-klinische Medizin mit diesem Problem weiter auseinander. Für ihn beruht (siehe die «Prolegomena» zum 2. Band der Medicina rationalis systematica) die «certitudo artis nostrae» auf der genauen Kenntnis des lebenden und gesunden Körpers, also auf der Physiologie; die «vera pathologia» habe zwei Fundamente, nämlich die auf striktester Beobachtung gegründete «historia» sämtlicher Krankheiten und die Kenntnis der Struktur und der Vorgänge im normalen Körper. Feinde der medizinischen «Sicherheit» seien die Hypothesen oder «opiniones» sowie die Fülle der verschiedenen «Sekten» (man erinnere sich an die gleichen Vorwürfe Agrippas). Ein wichtiges Problem der Physiologie ist auch für Hoffmann die Kenntnis gewisser «certi indices », vor allem derer des Todes.

Wenn man den langen Kampf um eine «Gewißheit der Heilkunde»oder was das gleiche ist: die Geschichte der medizinischen Skepsis - in ex-

15 Ich habe das von meiner Schülerin M. FENSCH in einer Dissertation Die monographische Behandlung des Themas ,Haar ${ }^{6}$ in der medizinischen Literatur des 18. und beginnenden 19. Jahrhunderts (Kiel 1965) untersuchen lassen.

${ }^{16}$ Ich beziehe mich hier auf die von mir angeregte Dissertation Scheintod als medizinisches Problem im 18. Jahrhundert von M.Augener, Kiel 1966. 
tenso darstellen will, wird man sich vor allem zweier Tatsachen immer bewußt bleiben müssen. Das eine sind die ungebrochene Tradition dieses Kampfes und die im Grunde immer gleichbleibenden Reaktionen auf eine immer gleiche geistige Konfliktsituation. Und das andere ist die Tatsache, daß es verschiedene Positionen in diesem Kampfe gibt, die in ihrer Physiognomie und ihrer Bedingtheit klar herausgearbeitet werden müssen. Das letztere haben wir hier in aller Vorläufigkeit an zwei charakteristischen Persönlichkeiten versucht. Die negative Position, bei uns markiert durch Agrippa von Nettesheim, müßte jetzt noch weiter gekennzeichnet werden. Auch im 16. Jahrhundert stand ja Agrippa nicht ganz allein, wenn wir nur an den Arzt-Philosophen Francisco Sanchez und seine Abhandlung «... quod nihil scitur» (Lyon 1581) denken. Auch der positive Standpunkt müßte ausführlicher umrissen werden; hier wäre etwa die Rolle Telesios im 16. und 17.Jahrhundert zu verfolgen. Ferner müßte untersucht werden, ob und wie sowohl die «Positivisten» wie die «Negativisten» zur Religion stehen, ob beispielsweise Boerhaaves Appell zur Erforschung der kleinen, ja der «verachteten» Dinge (Haut und Haar) mit seinem Pietismus zusammenhängt. Schließlich wäre die jeweilige Geltung der Mathematik und Geometrie als methodisches Modell bei den betreffenden Medizinern in Betracht zu ziehen. Genug, wenn wir hier fürs erste zwei gegensätzliche Positionen als solche fixiert und mit ihren Argumenten in einigen wesentlichen Punkten verdeutlicht haben. Mögen Berufenere hier weiterbauen. 